\title{
Business Model Adaptation: Evidence of Lean Experimentation in Digital Startups
}

\author{
Antonio Ghezzi \\ Politecnico di Milano \\ Antonio1.ghezzi@polimi.it
}

\author{
Silvia Sanasi \\ Politecnico di Milano \\ Silvia.sanasi@polimi.it
}

\author{
Angelo Cavallo \\ Politecnico di Milano \\ Angelo.cavallo@polimi.it
}

\begin{abstract}
Digital startups frequently adapt their business model, but in doing so they face resource scarcity and need to "make-do" in validating and implementing their design changes at a practical level. We thus argue that digital startups employ a Lean Experimental approach when adapting their $B M$ to contextual conditions. By means of an exploratory multiple-case study on Digital startups, this research investigates the factors driving the deployment of an experimental approach and proposing some factors that may drive differences in its application. Results suggest that most startups dealing with BM adaptation engage in experimentation practices that can be identified with the Lean Startup Approaches (LSAs), although with different extents of application. In this sense, startups move from scarce resource availability in resembling selected elements of the framework, whereas those with higher resource availability seem to be more prone to adopting LSAs in a structured and customized way at the organizational level.
\end{abstract}

\section{Introduction}

Most digital startups fail. The reason why often has to do with lack of resources and failure to adapt to the challenges posed by the environment [1]. This ability to adapt their strategy to uncertain conditions is strictly related to the capability of adapting their business model to the ever-changing needs of the context, as well as doing so with a very limited pool of resources [2]. In this sense, startups which eventually survive in their setting seem to deploy methods that help them overcome uncertainty, creating and capturing value in a more efficient way. These methods have an experimental basis and have been the object of several scholarly investigations in the most recent years $[3 ; 4]$. The continuous testing and assessment of the appropriateness of a given strategy involves the business model as the main object of experimentation. Business Models are defined as a company's architecture of value, and encompass the following main components: (i) value proposition (the ability to generate a benefit for target customers); (ii) value delivery (the ability to transfer those benefits to the market); (iii) target market (the customer segments targeted by the startup's value proposition) and (iii) value capture (the ability to reap a share of value and turn it into profits) [5]. Following this logic, business models are argued to be the result of several iterations and experimentation that entrepreneurs engage in.

However, the business model has only recently been addressed as the meeting point between entrepreneurship and strategy [2]: it is the means to combine advantage-seeking and opportunity-seeking behaviors. When designing business models, entrepreneurs define the boundaries of the business and the inherent value proposition to offer. This may be particularly complex and task-demanding, especially for technology-based ventures. Furthermore, the right business model rarely comes out at first attempts [5], but it is the result of extensive experimentation. Indeed, entrepreneurs must be ready to understand problems and consequently adjust their business model in parallel to the firm's evolution [5].

Experimental approaches are hence employed by established companies to innovate their business model. Similarly, the entrepreneurship literature generally argues that also startups implement this never-ending adaptation process through mechanisms of experimentation and learning. However, there is still no unified theoretical foundation concerning the approaches through which companies change their business model across their lifecycle.

We believe the understanding of the way digital startups build a sustainable and scalable business model by changing its dimensions is worth investigating. In fact, the theoretical and practical relationship between adaptation and one unified and shared method of experimentation lacks dedicated 
investigation. For these reasons, this study aims at exploring the way digital startups introduce changes in their business model and how this process of adaptation impacts on the different business model dimensions.

To implement such research, we carried out an exploratory multiple-case study to analyze the business model adaptation in digital startups acting on different value mechanisms [1]. The sample of analysis is composed of four startups operating digital businesses that differ in financing stage and number of employees: each of the startups underwent business model adaptation, each starting from a different value mechanism [5]. We investigated these ventures to understand (i) how these four startups changed their business model; as well as (ii) the method adopted by the startups to approach this process of change; and (iii) its impact on the different value mechanisms.

\section{Theoretical background}

The business model finds itself at the crossroads between strategy and entrepreneurship [2]: it is the architecture through which a firm creates, delivers and captures value from customers [5] and it is often the result of several attempts and iteration, i.e., experimentation. The concept has been developed since the 1990s, when the Internet boom forced companies to review their logics of value creation [6]. Despite a lack of clarity around the definition and the conceptualization of what a business model is, and the skepticism arisen among some strategy scholars who consider business model as "strategy in a new bottle", the awareness that it has become the new unit of analysis is well spread [6]. In fact, the business model is believed to play a key role in explaining firm performance [7], as well as a potential source of competitive advantage [6].

If the business model is a potential source of competitive advantage, designing the proper one is a crucial task. Nevertheless, business models may easily be subjected to modifications or adaptation.

Extant literature associates business model adaptation and change to different definitions and conceptualizations [8] such as business model evolution, renewal, learning [5], replication, erosion, lifecycle, transformation and innovation. The expression "business model innovation" is commonly used to refer to the literature streams concerning business model dynamics. However, Saebi et al. [1] distinguish between adaptation, i.e., a consequence of external factors, and innovation, i.e., which implies the voluntary change of an existing business model to disrupt market conditions and may be a consequence of either internal or external factors. Foss and Saebi [8] define business model innovation as the "novel, non-trivial changes to the key elements of a firm's business model and/or the architecture linking these elements" [8, p. 201].

Business model adaptation in all its forms can be a source of sustainable competitive advantage for both new ventures and incumbents [5]. However, the business model has been only recently addressed through an entrepreneurial lens. In fact, the primary goal of a startup is to find a viable business model, to generate value for customers and allows the startup to capture such value [4]. The literature stream dealing with entrepreneurial firms has been referred to as Strategic Entrepreneurship, and it interprets value creation through the process of discovery, creation and exploitation of opportunities [2].

There is a tight connection between business model and entrepreneurship. Entrepreneurs' choices mean, indeed, building hypotheses about what can be value for customers and how such value is created, delivered and captured [5]. Nevertheless, such process is far from being easy; especially the early phases might be highly complex and demanding, and this is particularly true for technology-based ventures operating in digital entrepreneurial ecosystems. Business model experimentation to rapidly test the market and validate business hypotheses is essential for entrepreneurial firms.

However, if the connection between business model adaptation and performance has been explored [7], the process through which entrepreneurial firms adapt their business model is still under scrutiny. Experimentation and learning have been depicted as essential elements to face business model adaptation and are often at the basis of systematic approaches e.g., Customer Development [9], Lean Startup [10] and Disciplined Entrepreneurship [11]. Recent contributions $[3 ; 4]$ propose to bundle these methods under the label of "Lean Startup Approaches" (LSAs), since they share goals - i.e. BM validation though scientific experimentation - and steps - i.e. hypothesizing, experiment design, testing, learning and pivoting of the original idea. Nevertheless, a clear understanding of a unified method to embark in business model adaptation has not been developed. We therefore argue that the understanding of how the process through which digital ventures reach a validated business model by changing its dimensions is worth the investigation.

\section{Methodology}




\subsection{Empirical setting}

The Italian hi-tech startup ecosystem has been gaining momentum in the last years, attracting a growing number of venture capital funds, business angels, incubators, accelerators, startups, and an increasing number of corporate venture capital funds. Politecnico di Milano's Hi-tech Startups Observatory report on the Italian startup ecosystem, has illustrated an unprecedented growth in equity funding destined to hi-tech startups: new ventures have raised 267 million euros more than in 2017 almost doubling the overall market value. This means the ecosystem has totaled almost 600 million euros of equity capital invested by both formal and informal investors. In particular, more than $30 \%$ of the investments come from international funds, also in this case doubling the previous year's balance. Such growing interest signals the presence of high-potential hi-tech startups are born and operate in the national market. As suggested by Isenberg [12], some of the factors influencing the thriving of an entrepreneurial ecosystem are highly-qualified human capital may be one. Furthermore, an increasing number of established companies are leveraging ideas and projects born in the entrepreneurial environment by collaborating with new creative and risk-taking ventures. This process of cross-fertilization and resource exchange keeps the ecosystem dynamic and alive. Given such increasing relevance, we therefore based our research on the Italian hi-tech startup context, focusing in particular on the process of business model adaptation in digital ventures.

\subsection{Materials and methods}

The research has been carried out as an exploratory multiple case-study $[13 ; 14 ; 15]$ which aims at analyzing how startups perform business model adaptation in a digital, dynamic, and disruptive context. Accordingly, we selected four ventures which underwent business model adaptation and operate in the digital contexts, where business model adaptation result to be frequent. Indeed, business model adaptation in digital startups is a contemporary and complex phenomenon fully embedded in its contexts, since the context's characteristics, such as environmental dynamicity, influence how it unravels.

We investigated the essence of a case study and the central tendency among all four case studies trying to inform a set of decisions: why they were taken, how they were implemented, and which results they obtained [13].
Being the case exploratory, no preordained relationship among variables and no specific proposition or hypothesis are anticipated, although we identified a theoretical contribution as a consequence of the empirical research performed [14]. In fact, even if exploratory cases should start with little or no theory and no hypotheses to test, it is impossible to begin researching with a "clear theoretical slate" [14]. Indeed, we started having in mind what business model design and business model innovation for entrepreneurial firms mean, as well as possible approaches to go through them. Nevertheless, we forced ourselves to be neutral using our theoretical background solely as a starting point to define the research question and set the data gathering process $[14 ; 15]$.

We therefore followed the Gioia methodology assumption of people as "knowledgeable agents" [16], without imposing any preordained knowledge. As Gioia et al. [16] argue, such approach paves the way for the discovery of new concepts rather the confirmation of existing ones which proves particularly appropriate as it supports our aim of generalization of the results.

In particular, we first followed an interpretive research approach, to understand the perspective of the people actually experiencing the events that have to be interpreted. Consequently, we interpreted the informants' voices through the lenses of business model adaptation theory, having in mind the possibility to develop new theory. Hence, after the interviews we linked the results to the extant theory on experimental approaches.

\subsection{Data gathering}

As the literature suggests $[13 ; 16]$, data was collected using multiple sources of information, comprising semi-structured interviews with selected informants, informal conversations, public presentations, and secondary sources (see Table 1 for further details). The aim of such comprehensive data gathering is to obtain both retrospective and real-time accounts from the people experiencing the phenomena [16]. Furthermore, we partly overlapped the data analysis and data collection phases so to introduce a more flexible collection of data. In fact, a key feature of theory-building case research is the freedom to make adjustments during the data collection process [14].

We implemented a process of triangulation of multiple sources of evidence in order to make the case study more reliable and accurate [13]. Before the interviews, we conducted research through secondary sources of information (e.g. company websites, 
company presentations, practitioner reports, etc.) to gather knowledge about each company in the sample. Then, we carried out semi-structured interviews as the primary source of evidence, so to stimulate open discussion and allow the emergence of issues that could not be thought in advance and that could reveal to be useful for the research's purpose [13].

The interviews involved the companies' founders and current employees. In addition to that, as Blue carried out a change in their business model at two different stages of their growth, a former employee was also included in the informants. We also conducted one pilot interview to test the clarity of the questions. We defined four sets of questions to ask the informants to describe how the business model adaptation, the process undertaken the key steps, methodologies and tools used.

\begin{tabular}{|c|c|c|c|}
\hline Data type & Quantity & $\begin{array}{l}\text { Original } \\
\text { data } \\
\text { source }\end{array}$ & $\begin{array}{l}\text { Original } \\
\text { (intended) } \\
\text { data } \\
\text { audience }\end{array}$ \\
\hline $\begin{array}{l}\text { Semi- } \\
\text { structured } \\
\text { interviews }\end{array}$ & $\begin{array}{l}1 \text { pilot } \\
\text { interview } \\
8 \text { company } \\
\text { interviews }\end{array}$ & $\begin{array}{l}\text { Informant } \\
\mathrm{s}\end{array}$ & $\begin{array}{l}\text { Analysis for } \\
\text { this study }\end{array}$ \\
\hline $\begin{array}{l}\text { Asynchronou } \\
\text { s } \\
\text { communicatio } \\
\text { n }\end{array}$ & $\begin{array}{l}19 \text { informal } \\
\text { emails }\end{array}$ & $\begin{array}{l}\text { Informant } \\
\mathrm{s}\end{array}$ & $\begin{array}{l}\text { Analysis for } \\
\text { this study }\end{array}$ \\
\hline $\begin{array}{l}\text { Documentatio } \\
\text { n }\end{array}$ & $\begin{array}{l}6 \\
\text { presentation } \\
\text { s } \\
2 \text { videos }\end{array}$ & $\begin{array}{l}\text { Informant } \\
\mathrm{s}\end{array}$ & $\begin{array}{l}\text { Public } \\
\text { presentation } \\
\text { s at } \\
\text { Politecnico } \\
\text { di Milano, } \\
\text { MIP School } \\
\text { of } \\
\text { Managemen } \\
\text { t, Talent } \\
\text { Garden } \\
\text { (Nexi POS } \\
\text { Revolution } \\
\text { Event) }\end{array}$ \\
\hline $\begin{array}{l}\text { External } \\
\text { documents } \\
\text { and sources }\end{array}$ & $\begin{array}{l}21 \text { internet } \\
\text { pages } \\
7 \text { newspaper } \\
\text { articles } \\
8 \text { Youtube } \\
\text { videos }\end{array}$ & $\begin{array}{l}\text { Informant } \\
\text { s } \\
\text { News } \\
\text { outlets }\end{array}$ & Public \\
\hline $\begin{array}{l}\text { Unstructured } \\
\text { interviews }\end{array}$ & $\begin{array}{l}4 \text { informal } \\
\text { conversatio } \\
\text { ns }\end{array}$ & $\begin{array}{l}\text { Informant } \\
\mathrm{s}\end{array}$ & $\begin{array}{l}\text { Analysis for } \\
\text { this study }\end{array}$ \\
\hline $\begin{array}{l}\text { Structured } \\
\text { Database }\end{array}$ & $\begin{array}{l}\text { Alba } \\
\text { Database, } \\
\text { containing } \\
\text { information } \\
\text { on } 801 \text { hi- } \\
\text { tech startups } \\
\text { and their }\end{array}$ & $\begin{array}{l}\text { Investors } \\
\text { Startups } \\
\text { News } \\
\text { outlets }\end{array}$ & $\begin{array}{l}\text { Politecnico } \\
\text { di Milano's } \\
\text { Hi-tech } \\
\text { Startups } \\
\text { Observatory } \\
\text { 's annual } \\
\text { research }\end{array}$ \\
\hline
\end{tabular}

funding

rounds from

2012 to

2019

Table 1. Sources of evidence employed.

\subsection{Data analysis}

The data was analyzed following Gioia et al.'s methodology [16], a holistic approach to inductive concept development which aims at bringing rigor to qualitative research. Through textual analysis, we firstly employed open coding to see which first order concepts were prevalent in the data. Then, we identified second-order themes, connected them to theoretical standpoints, and created a data structure from the cross-case analysis. Then, following the recommendations of Eisenhardt (1989), a within-case data analysis was carried out through Grounded Theory methodology $[17 ; 18]$. A subsequent crosscase analysis allowed us to make a comparison between the different responses given by the interviewees from the four startups. In particular, for each case we built an inductive coding tree following the "in vivo" procedure and also constructed codes [17]. Codes obtained from the interviews were iteratively compared to group them into sets of first order concepts. These first order concepts were then further grouped around a set of second order themes or categories, increasing the level of abstraction and facilitating our general understanding of concepts and data. Finally, the second order themes were grouped into overarching dimensions that captured the most important steps and constituting elements in a business model adaptation process. By means of these three-order analyses we have rigorously presented the connection between the data and the inductive concepts generated, to prove a high-quality qualitative study [16]. The data structure that results from these aggregated dimensions shows the process of abstraction starting from informants' codes to the last level dimensions [16].

With reference to cross-case analysis, we looked for similarities and differences between cases with reference to the first order concepts, second order themes and, above all, the overarching dimensions [13]. This concluding procedure allowed us to contrast and compare the adaptation process steps and methodology adopted within the four startups under investigation, allowing us to "capture the novel findings that may appear in the data" [14].

\section{Case description}


Four successful Italian startups operating in the digital ecosystem were selected as the object of the case study. Case sampling was performed theoretically [14] and based on heterogeneity. Specifically, the heterogeneity concerns the business model dimensions subjected to change - i.e. value proposition, value delivery, target market, value capture [1] -, and the startups' growth stage - i.e., number of employees and total amount of equity funding received. The industry diversity does not impact the results as all the four startups works in the hi-tech field; being digital, startups' changes could be applied to every field of application and business model modifications are not industry specific. Each of the startups selected for the analysis have been given imaginary names (i.e., Green, Blue, Red and Yellow) to preserve the privacy of the information shared.

\section{Findings}

As presented in the methodology section, each of the interviews was transcribed and then translated into a coding tree, aggregating interviews concerning the same case into the same coding tree. This inductive tool led us to draw the within-case discussion which, in turn, enabled the structuring of the final theoretical concepts. The use of these representations does not aim at defining a casual or dynamic model; it is instead a formal representation of the relationship between the direct results of the interviewees and abstracted concepts deriving from them.

More specifically, for all the four startups, the introductory phase of the interviews was centered upon a description of the process of adaptation in the introductory phase of the interviews. These first answers were always broad, and usually revealed those concepts that translated into the order themes concerning business model dimensions (e.g. value proposition, target market, value capture). Subsequently, the first, second, and third sets of questions refined the analysis by providing us with the missing content to draw a complete analysis of the change - i.e., which components were modified first and how they impacted on the whole business model. The fourth set of questions concerning the process, its phases, and the tools used for the adaptation, instead, inducted the coding of the concepts related to the overarching dimensions of "experimental approaches" and "lean principles". Finally, the "entrepreneurial behavior and innovative culture" concepts mainly originated from the introductive question and the fourth set.
It is worth noting that there is not always a straightforward connection between every question and the coded concepts. In fact, such concepts often derive from a combination of different answers to different questions. During and after the drafting of the coding trees, a cross-case comparison was performed to outline the similarities and the discordances among the different cases. This procedure allowed us to refine the abstraction process from the 1 st order concepts to the final overarching dimensions. We therefore drew a comparison among the four cases, on the basis of the coding trees outcomes. In particular, first order concepts, second order themes, and overarching dimensions were compared with the eventuality of finding any common pattern among the different cases. The cross-case analysis enabled the definition of the ultimate findings and the generation of the propositions concerning the connection between the process of adaptation, the maturity of a startup and the elements resulting from the coding trees.

\section{Discussion}

\subsection{Within-case findings}

6.1.1. Blue - change in value capture. Blue is operating in the digital marketing business. At the time of the change, September 2017, its total financing amounts were between 300 thousand and 500 thousand euros. The number of employees were around 30 people. The startup made two important changes in its three-years-long life. The second one was born in the attempt to scale, when Blue launched an ICO to receive funds. In fact, the structure of Blue was strictly similar to the one of the blockchain: decentralized, electronic payments with Coins and based on authorizations. As confirmed by the CEO: 'This is exactly the blockchain model. If we then think that the community where money is transacted are also rewarded with the same money, it is the equivalent of miners in the blockchain world. So, we saw the natural evolution of our model as it shares the main characteristics with the blockchain'.

They hence implemented a new revenue model, initially added to the existing one. The adoption of this form of payment also attracted new clients, that were familiar with the token environment and, thus, enlarged the customer base. Needless to say, the blockchain adoption implied the internal adjustment of activities and resources. Last but not least, by issuing its own tokens, Blue developed a complementary business model from which it can earn from the coins trading in the market. 
The startup is the case of a change in the Value Capture mechanisms. Nevertheless, the startup previously adapted the Value Proposition as well by introducing the gamification model, changing the Customer Relationship.

Both changes were declared to be the results of the Lean Startup Approaches application at a first sight. Thanks to the second round of interviews to a previous employee of the firm, it has been highlighted that the first change did not follow that approach step by step as much as the second one. The first change involved around 15 employees, which were not aware of the change was happening inside the firm and did not follow the different parts of the experimentation. In fact, decisions were on behalf of the founders and the build-measure-learn cycle did not appear as clear as in the second case, nor to the employees, nor to the founders.

Conversely, the Value Capture change invested all the 30 employees working in the startup, reshaping business units and creating a shared philosophy of decision making which characterizes the company vision and mission:

'For each decision, we are three decision makers: one that bring the community point of view - we are a two-sided platform, so we need to think about both sides -, one with the B2B clients' point of view; and one bringing the internal team point of view. We take decisions when we all agree.'

The Minimum Viable Product of the new platform is now in his trial phase on the website tokenbooster.com and will be available for final users as soon as the features are validated by future customers.

Another important issue the CEO highlighted is related to the application of the Lean Startup Approaches in presence of big partner such as Amazon: big companies do not want to fail, as it represents a reputational issue. For this reason, they do not like the presentation of the Minimum Viable Product on the market using their name and brand reputation, and it was one of the main problems during the phase of adaptation.

To conclude, the value capture dimension of Blue changes because users can buy the startup's coins and they will be able to use these coins to pay on the App. Moreover, part of the coins is owned by Blue; therefore, as much as users sell their digital money, the availability decreases and, consequently, their value increases. This implies that the Coins in Blue's pocket have a higher value, ensuring higher revenues. Blue's switch to this new revenue model has been successful because decisions were taken by future customers, those users that are interested in the cryptocurrency implementation and the same users that are raising the startup Coins' value.

6.1.2. Green - change in target market. The second case, Green, is a two years old startup operating in the field of artificial intelligence that changed the target market. At the time of the change there were more than 50 employees and the total financing amount were more than six million euros. Despite the good results some clients declared that the product was not working. The team comprehended that the problem was not the product itself, but the lack of enough data to make the system work. Business intelligence and machine learning mechanism need a huge amount of data to iterate and perfectionate the system capabilities. They realized the emergency of new clients, bigger than the former with a higher availability of data. The CEO decided to test the market applying a trust-me-I'm-lying approach: "You are supposed to tell everyone the product exists, you receive feedback and then you implement it as they want." The value proposition has remained the same, only some boundary features were adapted to the new target. The value delivery changed as they shifted from a one-to-all approach through a public website to a one-to-one tailored relationship where the product was released through the client's Intranet. They also changed the partners, that were previously identified in the big software vendors (e.g. Google, Facebook, Amazon). Subsequently to the change of target customer, Green needed to partner with system integrators having the ownership on the infrastructure of the startup's clients. Concerning the value capture dimension, the founder declared new cost items have been added in Green's cost structure, mainly due to legal and compliance issues, which is a peculiarity of bigger firms rather than SMEs. On the contrary, the revenue mechanism remained the leasing of the service.

Analyzing the aforementioned trust-me-I'm-lying approach, it is worth mentioning that that the CEO of the startup used this methodology to obtain a list of proof-of-concept, and the majority of the have become real projects. This latter approach is not the only strategy that led the company to a change in the business model, in fact Green has created a proper framework to face changes. The tool is composed of five pillars: intellectual property, team, company, partnership and product. These are the main dimensions of the change, the most relevant fields on which to focus to achieve the goal.

These pillars are alimented in parallel and each of them holds the same portion of attention.

"The framework became an asset during the fundraising phases, because we had a solid method. 
Then it allowed us to develop proprietary technologies."

Even if the declared methodology is 'learn and adjust', the startup did not rely on any known methodology or tool. The CEO mentioned the Lean Startup Approaches, as the name of its framework is 'the lean framework - how to avoid failure', but the main common object is the waste reduction principles. In fact, as the name suggests, there is not a failure culture inside the approach: "We adapted what we were doing using the proofs of concept. But the goal was to not fail as well as large enterprises do." The main reason why Green is trying to avoid failure is to not damage its reputation, especially with internal members.

6.1.3. Red - change in value proposition. The third case, Red, is a four years old startup, whose birth is due to the BlaBlaCar entrance on the market that pulled Bringme, a 2011-borned startup, out of the market. In fact, at the beginning Bringme dealt with long-distance trips, but, after the entry of that stronger foreign competitor, it was forced to renew its business model pivoting to Red. The value proposition of the business model pivoted to a new service for commuting needs of medium and big enterprises' employees: "Firms became our new customers. We created value for them by changing our value proposition." For Red this change has implied the adoption of new channels to deliver the offer and the internalization of some internal aspects concerning resources and activities. Red is the clear example of how a change of the value proposition led to the adaptation of the other dimensions.

The changing process started with an experimental project on September 2014, launching a free testing to two big companies and this phase lasted six months.

"Everything passed through testing. So, we got several feedback." When the founder was asked if any traditional approach was carried out, he answered: "I believe startups should use frameworks very little and rely on the founders' instinct. This is what happened. There is nothing scientific in the way we carried out the process." But asking more insight on the changing process he confirmed they used beta tests, hypotheses and market validation. Thanks to the beta test they started pivoting until the partnership with medium and big enterprises was defined. This is exactly the build-measure-learn cycle of the Lean Startup Approaches [10; 49].

At the time of the change Red had 14 employees and a total financing amount of four thousand euros.
6.1.4. Yellow - change in value delivery. The last case we examined was Yellow. It is a 2017-new born startup composed of the two founders and no other employees. At the time of the change the startup has received less than a hundred thousand euros of funding. Being an online marketplace, the app was only needed when the user was looking for a service from a professional. To incentivize the use of the app, the founders introduced Yellow Social, which is a social network where professionals can post about their activities. Users - i.e. those looking for the service - cannot post, but only interact with the activities posted by the other side of the platform. At the same time service provider will dispose of a section totally dedicated to statistics about their activity impact on the social network. By introducing the social network, Yellow changed the relationship with the customer, not affecting the value proposition. Only the App was adapted to the new features, while the website remained the traditional channel. Concerning the revenue model, Yellow keeps a royalty on the service equal to a percentage that varies on the amount.

Yellow is the case of a change of the value delivery dimension, which had no impact on the other dimensions of the business model and this phenomenon can be due to the small size of the company and also the simple process of implementation of the change. In fact, when we asked if they use some specific methodology, their answer was:

"We studied, taking inspiration from the most famous social networks. So, we have created a social halfway between Facebook, Instagram and even Google Plus." Therefore, they preferred to apply already validated successful business model rather than trying to create a tailored one. They used alpha and beta test, they had feedback and iteration, but the process was not the traditional one of the Lean Startup Approaches. They declared: "Sometimes you fall in love with your project, seeing only the positive aspects, but the Beta Test has given us some impartial indications for the operation." But they are too small to give birth to an iterative and continuous cycle of build-measure-learn. The MVP they presented was simple but almost complete of all the features of the final version.

\subsection{Cross-case findings}

First, we recognized that the process of adaptation is not relegated to the mere product or service innovation - which in this study is associated with the value proposition dimensions. This proves that 
entrepreneurs' focus has shifted from NPD to the wider concept of Business Model Innovation [5; 6].

Proposition 1. Business model validation and adaptation in digital startups does not only concern the Value Proposition but involves the overall business model.

In fact, all the four cases showed that the change of a startup business model may start from as well as consequently impact on the other dimensions of value delivery, creation and capture. We hence outlined the tight interconnection and interdependency among the value architecture dimensions, and how an adjustment of one implies the reconfiguration of the others. This first finding explains how the process of adaptation impacts on the overall business model and supports extant views [3; 4], who claim that an adjustment to one element of the business model entails an impact on the others.

Proposition 1.1 BM interdependencies determine that changing one dimension of the business model implies an impact on at least another dimension.

By proving such interdependency between different business model dimensions, this outcome questions the clear separation between modular and architectural changes, at least in the context of digital startups.

Second, we claim that experimentation is a constituent element of such process [20], extending the view of a simple facilitator of adaptation [1].

Proposition 2 The business model adaptation process in digital startups revolves around experimentation rather than planning.

The method of business experimentation involves the process of testing and concerns setting up business experiments. We argue that, in digital startups, this method is mainly deployed through the introduction of a Minimum Viable Product, further developed in a Minimum Viable Business Model (MVBM) in line with Proposition 1).

Proposition 2.1. The business model adaptation process in digital startups is based on experiments. The deployment of these experiments is often a Minimum Viable Product and a Minimum Viable Business Model.

However, the MVP and MVBM is used differently according to the stage of growth the startup finds itself in. In fact, at earlier stages, startups tend to create MVPs which incorporate more features simultaneously and tend to present them to a circumscribed circle of people - e.g., Yellow beta testing was addressed to few friends and developers and mainly used to add or slightly change product features. As the startup grows, MVPs are more loyal to their meaning of minimum amount of activities to disprove a hypothesis [19] and validated through customers feedback. Nevertheless, with startups at the later stage, the MVPs begins to be a more structured version, closer to MVBMs, in the sense that they incorporate the main concept but without boundary features, to satisfy business customers while reducing reputational risk.

Proposition 2.2. The notion and implementation of MVP depends on the growth stage of the startup.

This finding supports Eisenmann et al.'s [19] argument about the impact of an MVP on the reputation of the startup, while neglecting the authors' proposal to use a different brand to launch the MVP. In fact, Ghezzi [4] claims that the use of an "overly" minimum MVP is a consequence of dealing with business customers. However, experimentation is not solely related to product testing. Indeed, it has been shown evidence of testing on value capture, creation and delivery as well. This led us to argue that the overall business model change revolves around experimentation. This outcome also confirmed that new ventures follow a trial-and-error approach to continuously adapt their business model.

On the other hand, results from all four coding trees study also highlight that experimentation is often combined with customer involvement, continuous improvement and waste reduction to carry out adaptation. These findings draw a strong link between the use of experimentation in digital ventures and the lean philosophy.

Proposition 3. To reach business model adaptation, digital startups carry out a combination of experimentation, customer involvement, waste reduction, and continuous improvement principles.

These considerations led us to introduce the umbrella concept of Lean Startup Approaches [3; 4] in relation to the process of adaptation and the other elements of the analysis. This answered the research question investigating how digital ventures change their business model, also confirming the Lean Startup Approaches appropriate to validate not only a venture's value proposition, but all the elements of the business model [3]. 
Proposition 4. The overarching Lean Startup Approaches' principles are used to orchestrate the business model adaptation process in digital startups.

However, the fidelity and extent of application of the approach does not seem to be constant along the growth stages of the startup. As a matter of fact, our findings illustrate a trend of its use in relation to the stage of growth of the startup, pointing out that it low at lower level and it increases until the stage is higher, and the venture tend to abandon some practices as well as confine them to part of the organizations.

Proposition 4.1. The Lean Startup Approaches' extent of application during business model adaptation varies according to the startups' level of growth.

Furthermore, we related the extent of application of the Lean Startup to the mastery of use of the approach that the startups exhibited in the adaptation process. In particular, when startups have low mastery of entrepreneurial approaches tend to have limited application of Lean Startup, trying to imitate validated business models rather than following the structured model by Ries [10]. As the familiarity with Lean Startup increases, the application of the methodology rises, until the startup is able to customize it or even create a new one (e.g. Green).

Proposition 4.2. The Lean Startup Approaches' extent of application in business model adaptation varies according to the startup's mastery in the approach.

Irrespectively of the stage of growth, all the four cases presented a common element. A strong entrepreneurial behavior emerged indeed from the cross-case analysis, in the form of deploying entrepreneurial forms of organization, managing resources, applying creativity and bisociation to discover and exploit opportunities, exploiting founders' foresight and leadership. This permeated through the adaptation process of each startup, from the opportunity recognition to the vision of future reconfigurations.

Proposition 4.3. Entrepreneurial behaviors and innovative culture represent the foundation to recognize the need/opportunity for BMI, irrespectively of the stage of growth.
These findings support the perspective of organizational characteristics such as leadership, capabilities and learning as facilitator of adaptation [1].

In conclusion, the cross-case analysis has highlighted how the application of the LSAs is tied to the stage of growth of the startup, determined by the number of employees and the total amount of funding. We have noticed that, as the startup grows, the mastery of entrepreneurial approaches increases, causing the fidelity of the application of the LSAs to lower, in favor of personalization.

\section{Conclusions}

This study addresses the process that digital ventures undergo to adapt their business model as well as the impact of such change on the other dimensions of it. We implemented an explorative multiple case study on a sample of four startups that carried out adjustments on four different dimensions of their business model.

This research contributes to theory by tying emergent theory on business model experimentation to extant literature on entrepreneurship, demonstrating that the Lean Startup Approaches [3] can be perceived as a shared method to carry out changes on the whole business model in the context of digital entrepreneurial firms. Our study thus contributes to building theory on LSAs and sets the ground for future academic contributions in this direction. This research also sheds light on the tight interdependence between the different business model dimensions, hence questioning a clear separation between modular and architectural changes in the context of digital startups. We also propose a set of propositions that can be taken as reference for future contributions.

On a practical note, this research can serve as a guideline for managers and entrepreneurs in the implementation of LSAs when undergoing adaptation. The framework proposed can aid managers in identifying their growth stage and accordingly carrying out the correct approach for designing, validating and implementing changes in their company's Business Model.

The limitations of our study mainly refer to the biases related to the peculiarity of the context of digital startups and the size of the sample. These two factors may undermine the generalization and consequently the relevance of our findings. Future research should focus on validating the hypotheses made through a more comprehensive analysis based on a wide sample of respondents. 
A further weakness is related to the qualitative nature of our study and the inherent observer bias [13] that may potentially distort the informants' understanding of the questions as well as the researchers' interpretation of the answers. To overcome these limitations, we showed evidence of the suitability of the context of digital startups with the topic of adaptation. Moreover, we implemented a well-established method through the data gathering and analysis. Furthermore, the resultant theory is likely to be empirically valid because the theorybuilding process is so intimately tied with evidence to such an extent that the consistency between empirical observation and the resultant theory is reasonable. Nevertheless, further replication of our study on wider and different samples may reinforce the findings of this study.

\section{References}

[1] Saebi T, Lien L, Foss NJ. What drives business model adaptation? The impact of opportunities, threats and strategic orientation. Long range planning. 2017 Oct 1;50(5):567-81.

[2] Demil B, Lecocq X, Ricart JE, Zott C. Introduction to the SEJ special issue on business models: business models within the domain of strategic entrepreneurship. Strategic Entrepreneurship Journal. 2015 Mar;9(1):1-1.

[3] Ghezzi A, Cavallo A. Agile business model innovation in digital entrepreneurship: Lean Startup Approaches. Journal of Business Research. 2018.

[4] Ghezzi A. Digital startups and the adoption and implementation of Lean Startup Approaches: Effectuation, Bricolage and Opportunity Creation in practice. Tech. For. and Soc. Ch. 2019 Sept.; 146: 945-960.

[5] Teece DJ. Business models, business strategy and innovation. Long range planning. 2010 Apr 1;43(2-3):17294.

[6] Zott C, Amit R, Massa L. The business model: recent developments and future research. Journal of management. $2011 \mathrm{Jul} ; 37(4): 1019-42$.
[7] Zott C, Amit R. Business model design and the performance of entrepreneurial firms. Organization science. 2007 Apr;18(2):181-99

[8] Foss NJ, Saebi T. Fifteen years of research on business model innovation: how far have we come, and where should we go?. Journal of Management. 2017 Jan;43(1):200-27.

[9] Blank S. Why the lean start-up changes everything. Harvard business review. 2013 May 1;91(5):63-72.

[10] Ries E. The lean startup: How today's entrepreneurs use continuous innovation to create radically successful businesses. Crown Books; 2011.

[11] Aulet B. Disciplined entrepreneurship: 24 steps to a successful startup. John Wiley \& Sons; 2013 Jul 31.

[12] Isenberg D.J.. How to start an entrepreneurial ecosystem. Harvard Business Review. 2006

[13] Yin R. case study research. Beverly Hills. 1984.

[14] Eisenhardt KM. Agency theory: An assessment and review. Academy of management review. 1989 Jan 1;14(1):57-74.

[15] Eisenhardt KM, Graebner ME. Theory building from cases: Opportunities and challenges. Academy of management journal. 2007 Feb 1;50(1):25-32.

[16] Gioia DA, Corley KG, Hamilton AL. Seeking qualitative rigor in inductive research: Notes on the Gioia methodology. Organizational research methods. 2013 Jan;16(1):15-31.

[17] Glaser BG, Strauss AL. The discovery ofgrounded theory: Strategiesfor qualitative research. Chicago: Aldire. 1967.

[18] Strauss A, Corbin J. Basics of qualitative research techniques. Thousand Oaks, CA: Sage publications; 1998.

[19] Eisenmann TR, Ries E, Dillard S. Hypothesis-driven entrepreneurship: The lean startup. Harvard Business School Entrepreneurial Management Case. 2012 Mar 9(812-095).

[20] Sarasvathy SD, Venkataraman S. Entrepreneurship as method: Open questions for an entrepreneurial future. Entrepreneurship theory and practice. 2011 Jan;35(1):11335. 